\title{
STRICTLY REAL FUNDAMENTAL THEOREM OF ALGEBRA USING POLYNOMIAL INTERLACING
}

\author{
SOHAM BASU10
}

(Received 15 October 2020; accepted 10 November 2020; first published online 18 January 2021)

\begin{abstract}
Without resorting to complex numbers or any advanced topological arguments, we show that any real polynomial of degree greater than two always has a real quadratic polynomial factor, which is equivalent to the fundamental theorem of algebra. The proof uses interlacing of bivariate polynomials similar to Gauss's first proof of the fundamental theorem of algebra using complex numbers, but in a different context of division residues of strictly real polynomials. This shows the sufficiency of basic real analysis as the minimal platform to prove the fundamental theorem of algebra.
\end{abstract}

2020 Mathematics subject classification: primary 12D05; secondary 12D10, 26C10.

Keywords and phrases: fundamental theorem of algebra, polynomial interlacing.

\section{Introduction}

Let us consider a univariate polynomial of degree $N$ with its independent variable as well as fixed coefficients belonging to the field of real numbers $\mathbb{R}$, the smallest field containing all rational numbers and satisfying the least upper bound property [7]:

$$
f(x)=\sum_{n=0}^{N} c_{n} x^{n} \in \mathbb{R}, \quad x \in \mathbb{R}, c_{n} \in \mathbb{R} \text { for } 0 \leq n \leq N .
$$

The fundamental theorem of algebra (FTA), stated solely in terms of real numbers, asserts the factorisability of any such polynomial into a product of linear (degree one) and quadratic (degree two) polynomials with real coefficients [3]. Since $c_{0}=0$ forces $x$ to be a trivial factor, we restrict our consideration to $f(x)$ with $c_{0} \neq 0$. Ignoring trivial factorisations for $N \leq 2$, the FTA can be rephrased in a succinct version for $N \geq 3$.

THEOREM 1.1. For $N \geq 3$, given $c_{N}, c_{0} \neq 0$, there are $A, B \in \mathbb{R}$ and $d_{n} \in \mathbb{R}$ for $0 \leq n \leq N-2$ such that

$$
f(x)=\sum_{n=0}^{N} c_{n} x^{n}=\left(x^{2}-A x-B\right) \sum_{n=0}^{N-2} d_{n} x^{n} .
$$

(C) Australian Mathematical Publishing Association Inc. 2021. This is an Open Access article, distributed under the terms of the Creative Commons Attribution licence (http://creativecommons.org/ licenses/by/4.0/), which permits unrestricted re-use, distribution, and reproduction in any medium, provided the original work is properly cited. 
Even though the minimal algebraic elements for proving the FTA have been elucidated $[1,4,8]$, the minimal analysis platform to prove the FTA has not been characterised. We will show that the basic properties of the real number field suffice to prove Theorem 1.1 by considering the linear residue of the division of $\sum_{n=0}^{N} c_{n} x^{n}$ by $\left(x^{2}-a x-b\right)$ for $a, b \in \mathbb{R}$ :

$$
f(x)=\left(x^{2}-a x-b\right) q_{N-1}(x)+h_{N}(a, b) x+b h_{N-1}(a, b)+c_{0},
$$

which we will derive shortly. Both $h_{N}(a, b)$ and $b h_{N-1}(a, b)+c_{0}$ are bivariate polynomials in $a$ and $b$. By studying their interlacing [2], we will show that there are $A, B \in \mathbb{R}$ which satisfy $h_{N}(A, B)=0$ and $B h_{N-1}(A, B)+c_{0}=0$, thereby proving Theorem 1.1. The crucial steps will be to prove the following statements.

(1) There is $\bar{b}<0$ such that, for any fixed $b<\bar{b}, h_{N}(a, b)$ and $b h_{N-1}(a, b)+c_{0}$ have the maximum possible number of (real) roots in the variable $a$ which alternate with each other. For brevity, this property is abbreviated as interlacing.

(2) For some $b>\bar{b}$, interlacing fails either due to the failure in the alternating of the roots, or because $h_{N}(a, b)$ or $b h_{N-1}(a, b)+c_{0}$ has a smaller number of (real) roots than its degree in $a$.

Other strictly real proofs of the fundamental theorem of algebra (see [5, 6]) rely on advanced topological arguments.

\section{Establishing interlacing}

Directly checking for interlacing is intractable, so we resort to checking the interlacing of Sturm-like pairs $\left(h_{m-1}(a, b), h_{m}(a, b)\right)$ (see [9]), where $h_{m}(a, b)$ is the coefficient of $x^{N-m+1}$ at the $m$ th step of polynomial division of $f(x)$ by $x^{2}-a x-b$, as described below. Without initiating division,

$$
f(x)=\left(x^{2}-a x-b\right)(0)+c_{N} x^{N}+c_{N-1} x^{N-1}+\cdots+c_{0},
$$

which sets $q_{0}(a, b, x)=0, h_{0}(a, b)=0$ and $h_{1}(a, b)=c_{N}$. The first division step is to replace $x^{2}$ with $a x+b$ in the term with the largest power, $c_{N} x^{N}$, leading to

$$
\begin{aligned}
f(x)= & \left(x^{2}-a x-b\right)\left(c_{N} x^{N-2}\right) \\
& +\left(c_{N} a+c_{N-1}\right) x^{N-1}+\left(c_{N} b+c_{N-2}\right) x^{N-2}+c_{N-3} x^{N-3}+\cdots+c_{0} \\
= & \left(x^{2}-a x-b\right) q_{1}(a, b, x)+h_{2}(a, b) x^{N-1}+c_{N-3} x^{N-3}+\cdots+c_{0} .
\end{aligned}
$$

Next we divide the remaining term with highest power, $\left(c_{N} a+c_{N-1}\right) x^{N-1}$, to obtain the next term in the remainder:

$$
\begin{aligned}
f(x)= & \left(x^{2}-a x-b\right)\left(c_{N} x^{N-2}+\left(c_{N} a+c_{N-1}\right) x^{N-3}\right) \\
& +\left(c_{N} a^{2}+c_{N-1} a+c_{N} b+c_{N-2}\right) x^{N-2} \\
& +\left(c_{N} a b+c_{N-1} b+c_{N-3}\right) x^{N-3}+c_{N-4} x^{N-4}+\cdots+c_{0} \\
= & \left(x^{2}-a x-b\right) q_{2}(a, b, x)+h_{3}(a, b) x^{N-2}+\left(c_{N} a b+c_{N-1} b+c_{N-3}\right) x^{N-3}+\cdots+c_{0} .
\end{aligned}
$$


For larger $m$, we continue to show that

$$
\begin{aligned}
f(x)= & \left(x^{2}-a x-b\right) q_{m}(a, b, x)+h_{m+1}(a, b) x^{N-m} \\
& +\left(b h_{m}(a, b)+c_{N-m-1}\right) x^{N-m-1}+c_{N-m-2} x^{N-m-2}+\cdots+c_{0},
\end{aligned}
$$

with the following recursion formulae, which can be proved by induction:

$$
\begin{gathered}
q_{0}(a, b, x)=0, \quad h_{0}(a, b)=0, \quad h_{1}(a, b)=c_{N}, \\
q_{m+1}(a, b, x)=q_{m}(a, b, x)+h_{m+1}(a, b) x^{N-m-2}, \\
h_{m+2}(a, b)=a h_{m+1}(a, b)+b h_{m}(a, b)+c_{N-m-1} .
\end{gathered}
$$

Equations (2.1), (2.2) and (2.3) are the cases for $m=0,1$ and 2 respectively of the general equation (2.4). Equation (1.3) corresponds to $m=N-1$. An example of the scheme for a polynomial of degree eight is shown in Figure 1.

For $1 \leq m \leq N$ and fixed $b, h_{m}(a, b)$ is a polynomial in $a$ of degree $m-1$ and its leading term is $c_{N} a^{m-1}$. The other terms of $h_{m}(a, b)$ all have the form $c_{j, k} a^{j} b^{k}$ for some pairs $(j, k)$ and some nonzero constants $c_{j, k} \in \mathbb{R}$. Let $T_{m}$ represent the set of pairs $(j, k)$ for which $c_{j, k} a^{j} b^{k}$ other than $c_{N} a^{m-1}$ appears in $h_{m}(a, b)$. Then

$$
h_{m}(a, b)=c_{N} a^{m-1}+\sum_{(j, k) \in T_{m}} c_{j, k} a^{j} b^{k} .
$$

Equation (2.5) provides simple bounds for the roots of $h_{m}(a, b)$ in $a$ in terms of $b$.

LEMMA 2.1. Choose $C$ large enough such that $C \geq 1$ and $\left|c_{N}\right|>C^{-1} \sum_{(j, k) \in T_{m}}\left|c_{j, k}\right|$. Suppose that $b \leq-1$ and $1 \leq m \leq N$. If $h_{m}(a, b)=0$ for any such fixed $b$, then $|a|<$ $C \sqrt{|b|}$.

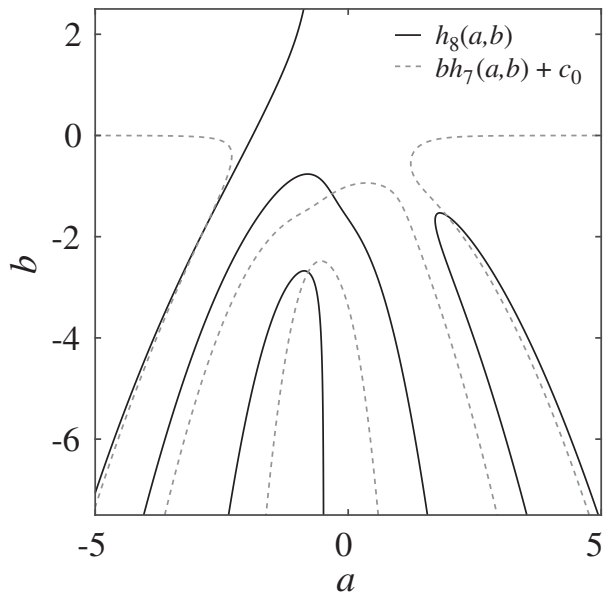

FIGURE 1. Illustration of $h_{8}(a, b)$ (solid black lines) and $b h_{7}(a, b)+c_{0}$ (dashed grey lines) corresponding to $f(x)=x^{8}+2 x^{7}+3 x^{6}+4 x^{4}+6 x^{3}+7 x^{2}+8 x+9$, exhibiting interlacing with maximum possible number of (real) roots in the variable $a$ for any fixed $b<-3$. The curves for $b h_{7}(a, b)+c_{0}=0$ never cross $b=0$. 
Proof. Suppose that $|a| \geq C \sqrt{|b|}$. Then

$$
h_{m}(a, b)=a^{m-1}\left(c_{N}+\sum_{(j, k) \in T_{m}} c_{j, k} \frac{b^{k}}{a^{m-1-j}}\right) .
$$

For any $(j, k) \in T_{m}$, we have $|a|^{m-1-j} \geq C^{m-1-j}(\sqrt{|b|})^{m-1-j} \geq C(\sqrt{|b|})^{2 k}=C|b|^{k}$ since $j \leq m-2$ and $j+2 k \leq m-1$. Consequently,

$$
\left|\sum_{(j, k) \in T_{m}} c_{j, k} \frac{b^{k}}{a^{m-1-j}}\right| \leq \sum_{(j, k) \in T_{m}}\left|c_{j, k}\right| \frac{|b|^{k}}{|a|^{m-1-j}} \leq \sum_{(j, k) \in T_{m}}\left|c_{j, k}\right| \frac{|b|^{k}}{C|b|^{k}} \leq \sum_{(j, k) \in T_{m}} \frac{\left|c_{j, k}\right|}{C}<\left|c_{N}\right|
$$

and

$$
\left|h_{m}(a, b)\right| \geq|a|^{m-1}\left(\left|c_{N}\right|-\left|\sum_{(j, k) \in T_{m}} c_{j, k} \frac{a^{j} b^{k}}{a^{m-1-j}}\right|\right)>0
$$

which completes the proof of the lemma.

LEMMA 2.2. Choose $\bar{b} \leq-1$ such that, for $0 \leq n \leq N$,

$$
\frac{\left|c_{N}\right|}{|\bar{b}|}+\frac{C\left|c_{N}\right|}{\sqrt{|\bar{b}|}}+\frac{\left|c_{n}\right|}{|\bar{b}|}<\left|c_{N}\right|
$$

If $b<\bar{b}, 2 \leq m \leq N$ and $h_{m}(a, b)=0$, then $\left|h_{m-1}(a, b)\right| \geq\left|c_{N}\right|$.

PROOF. Suppose that $\left|h_{m-1}(a, b)\right|<\left|c_{N}\right|$. We will prove that

$$
\left|h_{m^{\prime}}(a, b)\right|<\left|c_{N}\right| \text { for } 1 \leq m^{\prime} \leq m .
$$

The proof is by induction on $m^{\prime}$. If $m^{\prime}=m$, then (2.9) holds as $h_{m}(a, b)=0$ and, if $m^{\prime}=m-1$, then (2.9) holds by assumption. Suppose for some $m^{\prime}$ with $3 \leq m^{\prime} \leq m-1$ that $\left|h_{m^{\prime}}(a, b)\right|<\left|c_{N}\right|$ and $\left|h_{m^{\prime}-1}(a, b)\right|<\left|c_{N}\right|$. By the recurrence relation for $h_{m}(a, b)$ over $m$ and Lemma 2.1,

$$
\begin{aligned}
\left|h_{m^{\prime}-2}(a, b)\right| & \leq\left|\frac{h_{m^{\prime}}(a, b)-a h_{m^{\prime}-1}(a, b)-c_{N-\left(m^{\prime}-2\right)-1} \mid}{b}\right| \\
& \leq \frac{\left|h_{m^{\prime}}(a, b)\right|}{|b|}+\frac{C \sqrt{|b|}\left|h_{m^{\prime}-1}(a, b)\right|}{|b|}+\frac{\left|c_{N-m^{\prime}+1}\right|}{|b|} \\
& =\frac{\left|h_{m^{\prime}}(a, b)\right|}{|b|}+\frac{C\left|h_{m^{\prime}-1}(a, b)\right|}{\sqrt{|b|}}+\frac{\left|c_{N-m^{\prime}+1}\right|}{|b|} \\
& \leq \frac{\left|c_{N}\right|}{|\bar{b}|}+\frac{C\left|c_{N}\right|}{\sqrt{|\bar{b}|}}+\frac{\left|c_{N-m^{\prime}+1}\right|}{|\bar{b}|}<\left|c_{N}\right|,
\end{aligned}
$$

which completes the inductive proof of (2.9). However, for $m^{\prime}=3$, we have $\left|c_{N}\right|=$ $\left|h_{1}(a, b)\right|<\left|c_{N}\right|$, which is a contradiction. Therefore, Lemma 2.2 must hold. 
THEOREM 2.3. For all $b<\bar{b} \leq-1$ and all integers $m$ such that $2 \leq m \leq N$, the pairs $\left(h_{m-1}(a, b), h_{m}(a, b)\right)$ are interlacing in $a$. To recapitulate, for clarity:

(1) for fixed $b<\bar{b}, h_{m}(a, b)$ has $m-1$ distinct (real) roots in the variable $a$, which we designate by $\alpha_{1}^{(m)}(b)<\alpha_{2}^{(m)}(b)<\cdots<\alpha_{m-1}^{(m)}(b)$ in ascending order;

(2) the pairs $\left(h_{m-1}(a, b), h_{m}(a, b)\right)$ for $2 \leq m \leq N$ have interlacing roots, that is, $\alpha_{1}^{(m)}(b)<\alpha_{1}^{(m-1)}(b)<\alpha_{2}^{(m)}(b)<\cdots<\alpha_{m-2}^{(m-1)}(b)<\alpha_{m-1}^{(m)}(b)$.

PROOF. Fix $b<\bar{b} \leq-1$. We proceed by induction over $m$. Interlacing holds vacuously for the base case of $m=2$ as $h_{1}(a, b)$ has no roots. For the induction step, suppose that $3 \leq m \leq N$ and assume that the result holds for $m-1$, that is, $\left(h_{m-2}(a, b), h_{m-1}(a, b)\right)$ are interlacing. The number of roots of $h_{m-2}(a, b)$ is equal to its degree in $a$ and $h_{m-2}(a, b)$ changes sign at these roots. Therefore, the signs of $h_{m-2}\left(\alpha_{1}^{(m-1)}(b), b\right), h_{m-2}\left(\alpha_{2}^{(m-1)}(b), b\right), \ldots, h_{m-2}\left(\alpha_{m-2}^{(m-1)}(b), b\right)$ alternate. Moreover, the sign of $h_{m-2}(a, b)$ does not change for $a \geq \alpha_{m-2}^{(m-1)}(b)$. This implies that the sign of $h_{m-2}\left(\alpha_{m-2}^{(m-1)}(b), b\right)$ is the same as the sign of $h_{m-2}(a, b)$ for large $a$, which is the same as the sign of $c_{N}$ since the leading term of $h_{m-2}(a, b)$ is $c_{N} a^{m-3}$. Similarly, the sign of $h_{m-2}\left(\alpha_{1}^{(m-1)}(b), b\right)$ is the same as the sign of $c_{N}$ if $m-2$ is even and the opposite if $m-2$ is odd. Finally, by Lemma $2.1, \alpha_{l}^{(m-1)}(b)<C \sqrt{|b|}$ for all $l \in\{1,2, \ldots, m-2\}$.
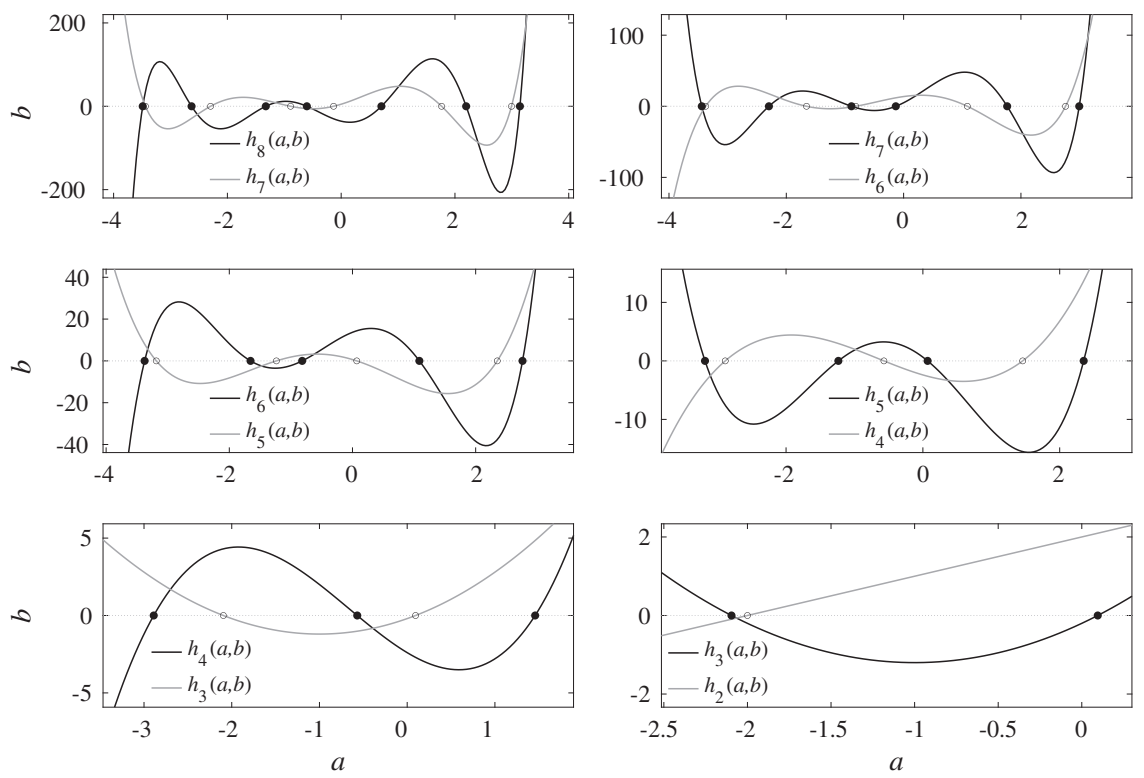

FIGURE 2. Illustration of interlacing of $\left(h_{m-1}(a, b), h_{m}(a, b)\right)$ in the variable $a$ for fixed $b=-3.2$ for the polynomial $f(x)=x^{8}+2 x^{7}+3 x^{6}+4 x^{4}+6 x^{3}+7 x^{2}+8 x+9$. 
By the recurrence for $h_{m}(a, b)$ and the definition of $\alpha_{l}^{(m-1)}(b)$, for $1 \leq l \leq m-2$,

$$
\begin{aligned}
h_{m}\left(\alpha_{l}^{(m-1)}(b), b\right) & =\alpha_{l}^{(m-1)}(b) h_{m-1}\left(\alpha_{l}^{(m-1)}(b), b\right)+b h_{m-2}\left(\alpha_{l}^{(m-1)}(b), b\right)+c_{N-m-3} \\
& =b h_{m-2}\left(\alpha_{l}^{(m-1)}(b), b\right)+c_{N-m-3} .
\end{aligned}
$$

The inequality (2.8) implies that $\left|c_{N-m-3}\right| /|b|<\left|c_{N}\right|$, which combined with Lemma 2.2 gives

$$
\left|b h_{m-2}\left(\alpha_{l}^{(m-1)}(b), b\right)\right| \geq\left|b \| c_{N}\right|>\left|c_{N-m-3}\right| .
$$

So, the sign of $h_{m}\left(\alpha_{l}^{(m-1)}(b), b\right)$ is the same as the sign of $b h_{m-2}\left(\alpha_{l}^{(m-1)}(b), b\right)$, which is the opposite of the sign of $h_{m-2}\left(\alpha_{l}^{(m-1)}(b), b\right)$. Also, when $|a|$ is sufficiently large, the signs of $h_{m-2}\left(\alpha_{l}^{(m-1)}(b), b\right)$ and $h_{m}\left(\alpha_{l}^{(m-1)}(b), b\right)$ agree. Therefore, an application of the intermediate value theorem establishes interlacing of $\left(h_{m-1}(a, b), h_{m}(a, b)\right)$.

Figure 2 illustrates the interlacing established in Theorem 2.3. Inequality (2.8) and Lemma 2.2 also imply that for $1 \leq l \leq N-1$ and $b<\bar{b} \leq-1$,

$$
\left|b h_{N-1}\left(\alpha_{l}^{(N)}(b), b\right)\right|>\left|b c_{N}\right|>\left|\bar{b} c_{N}\right|>\left|c_{0}\right| \text {. }
$$

So, $b h_{N-1}\left(\alpha_{l}^{(N)}(b), b\right)+c_{0}$ has the opposite sign to $h_{N-1}\left(\alpha_{l}^{(N)}(b), b\right)$. Applying the intermediate value theorem establishes interlacing for $\left(b h_{N-1}(a, b)+c_{0}, h_{N}(a, b)\right)$.

\section{Establishing $b h_{N-1}(A, B)+c_{0}=0$ and $h_{N}(A, B)=0$ from interlacing}

Proof of THEOREM 1.1. For $c_{0} \neq 0$, let us consider values of $b$ for which the pair $\left(b h_{N-1}(a, b)+c_{0}, h_{N}(a, b)\right)$ does not interlace. From the final remark in Section 2, this noninterlacing set is bounded below by $\bar{b}$. For small $|b|, h_{N-1}(a, b)=-c_{0} / b$ can only be attained either at two values of $a$ or none, due to the boundedness of the coefficients of $h_{N-1}(a, b)$ when written as a polynomial in $a$. In particular, when $b=0$, $b h_{N-1}(a, b)+c_{0}=c_{0}$ is constant and therefore has no roots. Thus, the noninterlacing set contains some interval around $b=0$. Since the noninterlacing set is nonempty and bounded below, by the least-upper-bound property [7] it has an infimum $B \in \mathbb{R}$ satisfying $\bar{b} \leq B<0$. Since the coefficients of both $h_{N}(a, b)$ and $b h_{N-1}(a, b)+c_{0}$ as polynomials in $a$ are bounded for $\bar{b} \leq b \leq B<0$, the roots of both $h_{N}(a, b)$ and $b h_{N-1}(a, b)+c_{0}$ in the variable $a$ are bounded for these values of $b$. Thus, by the Bolzano-Weierstrass theorem, roots of $h_{N}(a, b)$ and $b h_{N-1}+c_{0}$ can be defined at $b=B$ as subsequential limits of their roots taken over any sequence $b_{k}$ of terms such that $\left\{b_{k}: k \in \mathbb{N}, \bar{b} \leq b_{k}<B, b_{k}<b_{k+1}, \lim _{k \rightarrow \infty} b_{k}=B\right\}$. For $b<B$, writing the roots of $b h_{N-1}(a, b)+c_{0}$ as $\beta_{l}(b)$ for $1 \leq l \leq N-2$, we can define limits over any converging subsequences of $\beta_{l}\left(b_{k}\right)$ and $\alpha_{l}^{(N)}\left(b_{k}\right)$ indexed by $\left\{\sigma_{k}: k \in \mathbb{N}\right\}$ :

$$
\begin{gathered}
\beta_{l}=\lim _{k \rightarrow \infty} \beta_{l}\left(b_{\sigma_{k}}\right), \quad 1 \leq l \leq N-2, \\
\alpha_{l}=\lim _{k \rightarrow \infty} \alpha_{l}^{(N)}\left(b_{\sigma_{k}}\right), \quad 1 \leq l \leq N-1 .
\end{gathered}
$$

Applying $\alpha_{1}^{(N)}\left(b_{\sigma_{k}}\right)<\beta_{1}\left(b_{\sigma_{k}}\right)<\alpha_{2}^{(N)}\left(b_{\sigma_{k}}\right)<\cdots<\beta_{N-2}\left(b_{\sigma_{k}}\right)<\alpha_{N-1}^{(N)}\left(b_{\sigma_{k}}\right)$ gives

$$
\alpha_{1} \leq \beta_{1} \leq \alpha_{2} \leq \cdots \leq \beta_{N-2} \leq \alpha_{N-1}
$$


If all the inequalities are strict, then $B$ belongs to the interlacing set. Then there exists $t>0$ such that $\left(\alpha_{i}-t, \alpha_{i}+t\right)$ and $\left(\beta_{j}-t, \beta_{j}+t\right)$ are disjoint. This implies that $h_{N}\left(\alpha_{i}-t, B\right) h_{N}\left(\alpha_{i}+t, B\right)<0$ and $\left(B h_{N-1}\left(\alpha_{j}-t, B\right)+c_{0}\right)\left(B h_{N-1}\left(\alpha_{j}+t, B\right)+c_{0}\right)<0$. From the continuity of bivariate polynomials, there exists $\delta$ with $0<\delta<-B / 2$ such that $h_{N}(a, b)$ and $b h_{N-1}(a, b)+c_{0}$ do not change sign inside circles of radius $\delta$ centred around $\left(\alpha_{i} \pm t, B\right)$ and $\left(\beta_{j} \pm t, B\right)$, respectively. Since $h_{N}\left(\alpha_{i}-t, b\right) h_{N}\left(\alpha_{i}+t, b\right)<0$ for $B \leq b<B+\delta$,

$$
h_{N}(a, b) \text { has at least one root in }\left(\alpha_{i}-t, \alpha_{i}+t\right) \text { for } B \leq b<B+\delta
$$

and, since $\left(b h_{N-1}\left(\beta_{j}-t, b\right)+c_{0}\right)\left(b h_{N-1}\left(\beta_{j}+t, b\right)+c_{0}\right)<0$ for $B \leq b<B+\delta$,

$$
b h_{N-1}(a, b)+c_{0} \text { has at least one root in }\left(\beta_{j}-t, \beta_{j}+t\right) \text { for } B \leq b<B+\delta .
$$

But then interlacing holds for $B \leq b<B+\delta$, which contradicts the definition of $B$ as the infimum of the noninterlacing set. Therefore, at least two neighbouring quantities in (3.1) must be equal. The value of $A$ is given by these equal quantities at $b=B$, thereby proving Theorem 1.1 .

Additionally, we have proven that we can always find $B<0$ satisfying Theorem 1.1. To obtain the complete factorisation of $f(x)$, Theorem 1.1 can be applied onward from $\sum_{n=0}^{N-2} d_{n} x^{n}$ until it halts due to exhausting all powers of $x$.

\section{References}

[1] M. Aliabadi, 'A note on the fundamental theorem of algebra', Bull. Aust. Math. Soc. 97(3) (2018), $382-385$.

[2] S. Basu and D. J. Velleman, 'On Gauss's first proof of the fundamental theorem of algebra', Amer. Math. Monthly 124(8) (2017), 688-694.

[3] C. F. Gauss, Demonstratio Nova Theorematis Omnem Functionem Algebraicam Rationalem Integram Unius Variabilis In Factores Reales Primi Vel Secundi Gradus Resolvi Posse, PhD Thesis, Helmstedt, 1799.

[4] D. Perrucci and M. F. Roy, 'Quantitative fundamental theorem of algebra', Q. J. Math. 70(3) (2019), 1009-1037.

[5] A. V. Pukhlikov, "Real" Proof of the Fundamental Theorem of Algebra, Matematicheskoe Prosveshchenie Series 3, 1 (Moscow Center for Continuous Mathematical Education, Moscow, 1997), 85-89 (in Russian).

[6] P. E. Pushkar, On Some Topological Proofs of the Fundamental Theorem of Algebra', Matematicheskoe Prosveshchenie Series 3, 1 (Moscow Center for Continuous Mathematical Education, Moscow, 1997), 90-95 (in Russian).

[7] W. Rudin, Principles of Mathematical Analysis, 3rd edn (McGraw-Hill, New York, 1976), Sect. 1.10.

[8] J. Shipman, 'Improving the fundamental theorem of algebra', Math. Intelligencer 29 (2017), 9-14.

[9] J. C. F. Sturm, 'Mémoire sur la résolution des équations numériques', Bull. Sci. Férussac 11 (271) (1829), 419-425.

\section{SOHAM BASU, School of Engineering,}

École Polytechnique Fédérale de Lausanne, Lausanne 1015, Switzerland e-mail: sohambasu6817@gmail.com 